\title{
Das Hospiz ist fern - gestorben wird daheim
}

\author{
Experten fordern die Hausärzte auf, Menschen am Ende des Lebens vor Überversorgung zu bewahren \\ (siehe Interview ab S. 16). Wie das funkionieren kann, zeigt eine Landarztpraxis in Niedersachsen.
}

\begin{abstract}
- Richtig gute Ideen passen auf einen Bierdeckel. So wie jene der Hausärzte Michael Pohling, Carla und Dr. Ekkehard Martin aus Lehre bei Wolfenbüttel, deren Modell schon vielen todkranken Patienten und ihren Angehörigen geholfen hat. Denn die Patienten müssen gar nicht mehr ins Krankenhaus gehen.
\end{abstract}

Zusammen mit Pflegekräften, die für die spezialisierte ambulante Palliativversorgung (SAPV) ausgebildet sind, und dem in Palliative Care ausgebildeten Team des Alten- und Pflegeheimes „Haus Wartburg" versorgen sie sterbende Patienten wie in einem Hospiz - aber zu Hause bzw. im Heim. Damit haben sie das Rad nicht neu erfunden, aber seine Bestandteile neu zusammengesetzt.

Kompetenz auf pflegerischer und ärztlicher Seite war vorhanden, die Räume im Heim auch. Die zündende Idee war, beides zu kombinieren. Das „gemeindeorientierte Palliativnetz" funktioniert sehr gut, sagen die drei Ärzte. Vor allem die Pflegenden übernehmen die Versorgung. Von den Ärzten haben sie einen „Palliativkrisenbogen“ bekommen mit den Versorgungsschritten bei Angst, Unruhe, Krämpfen oder Schmerzen. „Die Arbeit wird uns dadurch enorm erleichtert, und den Patienten ist gedient", sagt Pohling.

\section{Eine Eingebung des Moments}

Die Idee entstand, da weder ein Hospiz noch eine Palliativstation in der Nähe sind. 2014 überwies Pohling einen $\mathrm{Pa}$ tienten kurzerhand ins Pflegeheim Wartburg und setzte bei der Krankenkasse durch, dass sie die Transportkosten übernahm. So konnte der Mann im Pflegeheim palliativ versorgt werden. Warum nicht immer diesen Weg beschreiten?

Heute begleiten die Ärzte Sterbende wohnortnah und umgeben von vertrau-

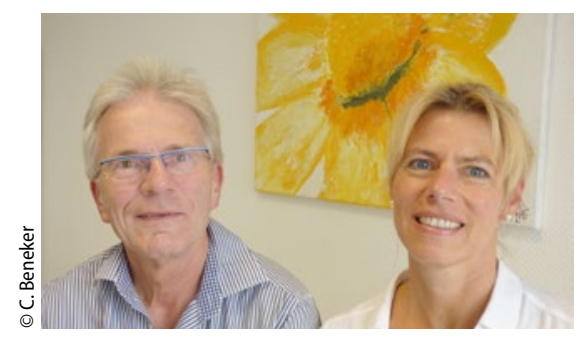

Die Hausärzte Pohling und Martin.

ten Menschen. Inzwischen fragen auch weiter entfernte Krankenhäuser bei den Ärzten an, um ihre Palliativpatienten nicht in eine möglicherweise schwierige Pflegesituation etwa nach Hause entlassen zu müssen. Derzeit versorgen Ärzte und Pflegende jährlich rund 25 Palliativpatienten im Pflegeheim.

Die Idee braucht nun einen tragfähigen Vertrag mit den Kassen, meinen die
Ärzte. „Es sollte einleuchten, dass man mit unserem Projekt eine Menge Geld sparen kann“, sagt Martin. Immerhin müssen die Kassen im Hospiz 95\% der Kosten übernehmen. Im Haus Wartburg müssen die Angehörigen wie für jeden Pflegeplatz zwischen 1.252 und 1.551 Euro im Monat hinzubezahlen.

Pohling und Martin können sich für die Finanzierung ein Modellprojekt nach $\$ 72$ SGB XI vorstellen, um den gleichen Status wie ein Hospiz zu erhalten. „Vor allem die Transportkosten von zu Hause ins Pflegeheim müssten die Kassen übernehmen“, fordert Pohling. Außerdem brauchen die Pflegenden im Heim mehr Geld. „Für uns Ärzte zählt in dem Projekt nicht höheres Honorar", sagt Pohling, „sondern vor allem die Arbeitserleichterung“.

Christian Beneker

\section{Erfolgs-Rezept - der Praxis-Preis 2016}

\section{Bewerben Sie sich jetzt mit Ihren Ideen für die Praxis!}

Seit fünf Jahren loben UCB Innere Medizin und Springer Medizin unter dem Namen „Die innovative Arztpraxis" den Praxis-Preis für Ärzte mit größeren oder kleineren Ideen für eine bessere Versorgung oder für eine effiziente Praxisführung aus.

Haben Sie eine Idee umgesetzt, die Ihnen hilft, den Praxisalltag zu verbessern? Wissen Sie, wie Sie Ihre Patienten strukturiert versorgen können? Können Sie die Kommunikation im Praxisteam oder mit anderen Praxen oder Einrichtungen optimieren? Oder haben Sie vielleicht eine kleine Idee entwickelt, die Ihre Praxis voranbringt, an die noch niemand gedacht hat?

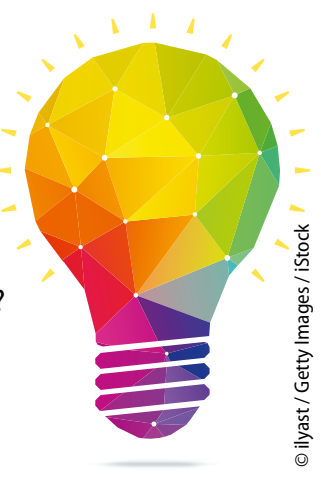

Dann machen Sie mit beim diesjährigen Wettbewerb „Erfolgs-Rezept - der Praxis-Preis 2016“! Ärzte und Praxisteams können sich bis zum 30. November anmelden. Unter www. aerztezeitung.de/erfolgsrezept finden Sie dafür ein Formular.

Eine unabhängige Jury und die Nutzer von aerztezeitung.de küren am Ende drei Gewinnerteams, die ihre Konzepte bei Springer Medizin in Berlin persönlich vorstellen. Zu gewinnen gibt es einen dreitägigen Ausbildungslehrgang mit Abschluss "Geprüfte Assistenz für Versorgung und Prävention" von der Unternehmensberatung HCC Better Care GmbH, Köln, sowie Buchpreise von Springer Medizin und HCC.

- ger/mh 